\title{
Translucent Root Dentine in Relationship to Increasing Age: Review of the Literature
}

\author{
${ }^{1}$ L. Vasiliadis, ${ }^{1}$ C. Stavrianos, ${ }^{2}$ P. Dagkalis, ${ }^{1}$ Ks. Parisi, ${ }^{1}$ I. Stavrianou and ${ }^{1}$ D. Tatsis \\ ${ }^{1}$ Department of Endodontology (Forensic Odontology), \\ ${ }^{2}$ Department of Preventive Dentistry Periodontology and Implant Biology, \\ School of Dentistry, Aristotle University, Thessaloniki, Greece
}

\begin{abstract}
Different methods have been proposed in the course of time of age estimation via human biological characteristics. One of them is the measurement of dentine translucency. The amount of transparent dentine that is deposited through time is strongly related with the age of the person. Some controversy on the accuracy of the method has been stated, thus it is evident by numerous studies that the sclerotic dentin that is formed during aging due to altering rates of mineralization has successfully lead in age estimation of experimental subjects. Finally, the results of the past studies are coherent with the current literature, proving that age estimation via root dentine translucency is possible in clinical basis.
\end{abstract}

Key words: Dentine translucency, age determination, endodontics, forensic odontology, mineralization, Greece

\section{INTRODUCTION}

The translucency of dentine which may be present either as zones of translucent dentine which occur as a result of external irritations or as the translucent dentine of the root which occurs with increasing age is a topic that has attracted the interest of many researchers in the past. Despite this interest, present knowledge about the translucency of root dentine is far from being complete as the findings of researchers on this subject are often in disagreement and the theories proposed to explain the reasons and mechanisms of its formation are not universally accepted.

During what can be called the 1st stage of research (1850-1940), the main fields of study were the staining properties and permeability of the dentine. This resulted in the finding that the changes that make the dentine appear translucent occur in the lumen of the dentinal tubules. Understandably, this also gave rise to greater confusion between translucent dentine and what are known as dead tracks because both are at least partly impermeable to dyes from the pulp (Beust, 1931a-c, 1934). The reason that the dentine becomes translucent was assumed to be the occlusion of the dentinal tubules with some substance having the same or almost the same refractive index as the rest of the dentine which results in the development of an optically homogeneous dentine (Fish, 1933; Manly et al., 1940). With the development of more refined chemical analytical techniques and the introduction of microradiography (1940 onwards), new findings were reported which were often contradictory. From the findings of chemical analysis, it was concluded that the material occluding the lumens of the dentinal tubules of the translucent areas of the root dentine was mineralized to the same extent as the cementum (Simon and Armstrong, 1941; Manly and Brooks, 1947). Micro radiography showed that it was mineralized to the same degree as peritubular dentine (Nalbandian et al., 1960; Isokawa et al., 1966; Takuma and Eda, 1966). Microhardness examination, the 3rd examination method employed during this stage gave inconsistent results and did not help to solve the problem.

At the present time, although finer tools are available TEM, SEM, electron probe analysis, etc., the disagreement about the exact nature of the occluding material still exists. Some of the past arguments are still unresolved and there is still no convincing explanation for its formation. The zones of translucent dentine under external irritation are considered to be the attempts of the pulpo-dentinal complex to protect it self from the external irritants by blocking off the dentinal tubules involved. It is generally accepted that the translucency of the root dentine increases in a linear manner with age (Gustafson, 1947; Bang and Ramm, 1970; Johanson, 1971; Azaz et al., 1977), although there is no universally accepted explanation for the reasons of its formation. Toxins from diseased periodontal tissues (Miles, 1976), negative mastication pressures and the smaller diameter of the dentinal tubules of this area (Bradford, 1960) have all been advanced as reasons for its appearance.

Corresponding Author: L. Vasiliadis, Department of Endodontology (Forensic Odontology), School of Dentistry, Aristotle University, Thessaloniki, Greece 
The amount and distribution of the translucent root dentine and its relationship to increasing age: Gustafson (1947, 1950) was the 1st investigator to make use of the fact that the root dentine of human teeth becomes translucent with age. He held the opinion that this translucency is not closely related to pathological conditions and added the criterion to the already existing ones used for the age determination of teeth for forensic purposes. He studied the distribution and estimated the amount of the translucent dentine of the root by optical examination of ground longitudinal sections, through the pulp prepared by grinding the two sides of the tooth. $\mathrm{He}$ found that translucency starts at the apex and extends gradually towards the cervical area of the root. There is a definite increase in the amount of translucent dentine with increasing age. He does not offer any information about the thickness of the sections which he examined.

Miles (1961) states that although the extent of root dentine translucency is at least loosely correlated with age, it is by no means certain that it represents an intrinsic age change. Contrary to Gustafson (1950), he seems to favour the belief that root dentine translucency is closely associated with pathological conditions, namely the effect of toxins from diseased periodontal tissues on the odontoblasts of this area. In more recent publications, Miles $(1972,1976)$ states that from the changes which are used in assessing the age for forensic purposes, translucency of the root apex seems to be the most reliable or the one with the closest straight-line relationship with age. It seems that in a fairly orderly way, there is a gradual extension of the processes responsible for this translucency further and further in the direction of the crown as age advances until in due course, the whole of the root may be affected. Johnson (1968) examined the relation between age and the amount of translucent dentine in the roots of human teeth of known age. $\mathrm{He}$ used single-rooted teeth which were ground in a labio-lingual plane, so that a complete section of the root and the crown remained. He reports that his results show that the measurement of the areas of translucent and opaque root dentine is not a suitable method on which to base the estimation of the age of the tooth donor. In other words, he was unable to find a good correlation between the area of translucent dentine and age. Bang and Ramm (1970) firstly examined the roots of intact teeth with the naked eye, measuring the total length of the root and the distance between the apex and the borderline between translucent and opaque dentine. At a later stage, Bang and Ramm examined ground longitudinal sections cut at the middle of the root. The image of the each section was projected onto paper and the area of total dentine and translucent dentine were outlined and then measured.

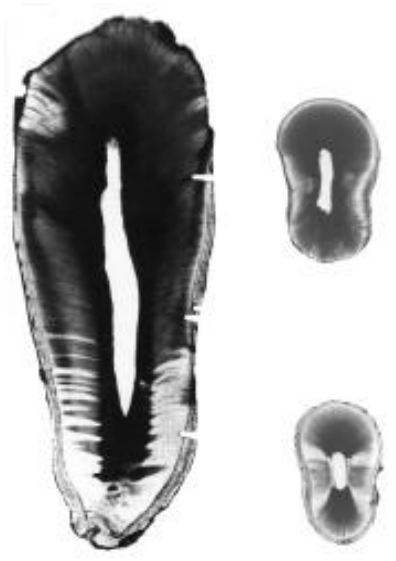

Fig. 1: Left) Longitudinal section, 255-270 $\mu \mathrm{m}$ thick from canine sample, age 57 . Note the pattern of spread from the cemento-dentinal junction and frequent occurrence of zones of normal dentine interspaced with the zones of translucent dentine (mag. x9.5); Right) Cross sections of the roots as examined by the enlarger technique. Male, age 60 , lower right canine $(\mathrm{A}=$ cervical third, $\mathrm{B}=$ middle third). Note the butterfly shape of the translucent zones and the relative increase in translucency with age and as the apex is approached (mag. $\mathrm{x} 8$ ) (Vasiliadis et al., 2008)

They report that their results showed that there is a significant increase in root transparency with age. Also that the results from both methods are acceptable as criteria on which the age estimation of the tooth donor may be based. From the way, the length of the translucency was measured, it has been assumed that they found translucency of the root dentine to start at the apex of the tooth and to extent gradually more coronally with age (Fig. 1).

Johanson (1971) tried to evaluate and improve Gustafson's method of age estimation from human teeth. He examined photographic enlargements of bucco-lingual or bucco-palatal longitudinal ground sections, $250 \mu \mathrm{m}$ thick through the pulp. Results show that from the 6 criteria used in Gustafson's method, the one least affected by pathological conditions and which gives the best correlation with age is the amount of translucent dentine of the root. He too reports that translucency always starts near the apex and spreads with age towards the cementoenamel junction.

Azaz et al. (1977) studied the amount of translucent root dentine and cementum of totally impacted human canines using longitudinal labiolingual ground sections, $250 \mu \mathrm{m}$ thick. They report that the relative areas of translucent dentine and cementum are shown to increase 
linearly with age. The totally impacted canines used in their investigation were subject to external irritation and the above described changes are due to age only.

\section{DISCUSSION}

Tomes was the first investigator to describe translucent dentine. He wrote that translucency is the result of the consolidation of the dentinal tubes and he noticed that if the air in the dentinal tubules of a tooth is replaced by water the tooth becomes more translucent (Vasiliadis, 1981). He and Czermak held the opinion that various types of translucency arose because of an equalization of the normally different indices of refraction of the tubules and of the calcified dentine matrix. Fish (1933) believed that transparency is the result of an increase in the optical homogeneity of the dentine.

It is commonly accepted that translucent dentine appears translucent because instead of refracting, reflecting and scattering the light as the normal dentine does, it allows the light to pass through it. This explanation is supported by the findings that translucency of the dentine is associated with a decrease in the light scattering as indicated by a lessened depolarization of light (Hodge, 1937) and by a fall of over $95 \%$ in the scattering coefficient (Manly et al., 1940). The translucency of the dentine will increase with changes which decrease the size or the number of refracting surfaces within the dentine or the amount of refraction per surface.

Of the three main components of dentine, i.e., apatite crystallites, collagen fibrils and tubules, only the tubule diameter is greater than the wavelength of light and therefore the tubules must be the source of the refraction and scattering of the light. Consequently if the above statements are correct, any changes that result in the decrease of the size or the number of the dentinal tubules or the amount of refraction per tubule must increase the transparency of the dentine.

The results of different examination methods have shown that the lumens of the dentinal tubules in the translucent dentine are partly or completely closed. Manly and Brooks (1947) measured the amount of light passing through areas of dentine sections before and after replacing the contents of the dentinal tubules with media having different refracting indices.

They found that the amount of the transmitted light is fairly steady for areas of the dentine which are translucent in dry ground sections when measured with a photoelectric cell attached to a microscope. For areas of opaque, normal dentine they found that transparency reaches its maximum when the tubules are filled with media having refractive indices very close to that of the rest of the dentine or in other words when the amount of refraction per surface falls to a minimum.

\section{CONCLUSION}

Estimating of a human corpse age is a significant part of forensics during the identification process of a cadaver that cannot be identified otherwise due to denaturation of the individual characteristics that would lead to an optical recognition. Furthermore, the age estimation is crucial during law enforcement tasks as living individuals without registered identification can be classified as adults or minors and can be treated by the law agencies likewise. All the scientists and researchers that are working on human age identification have come up with different methods to do so such as developmental, histological, biochemical and anthropological techniques. Anthropologists for example, analyze the fusion of the cranial sutures of the skull, the development of the long bones, features of the pelvic girdle in order to estimate the age of the deceased. Odontologists on the other hand, study the features of teeth in order to conclude safely on the age. The methods of age estimation using teeth include analyzing tooth development and eruption, studying tooth degradation and measuring biochemical and trace element changes in dental structures. All of these methods have certain advantages and limitations concerning the accuracy, the cost-effectiveness, the difficulty of use, etc. The appropriate method is selected primary according to the examiners' skill and available technical equipment (radiographic equipment, examination laboratory, etc.) (Harris et al., 2010).

\section{REFERENCES}

Azaz, B., Y. Michaeli and D. Nitzan, 1977. Aging tissues of the roots of nonfunctional human teeth (impacted canines). Oral Surg. Oral Med. Oral Pathol., 43: 572-578.

Bang, G. and E. Ramm, 1970. Determination of age in humans from root dentin transparency. Ácta Odintol. Scand., 28: 3-35.

Beust, T.B., 1931a. Reactions of the dentinal fibril to external irritation. J. Am. Dent. Assco., 18: 1060-1073.

Beust, T.B., 1931b. Physiological changes in the dentine. J. Dent. Res., 11: 267-275.

Beust, T.B., 1931c. Posteruptive changes in the maturation of teeth. J. Am. Dent. Assco., 18: 2186-2192.

Beust, T.B., 1934. Demonstration of sclerosis of dentine in tooth maturation and caries. D. Cosmos, 76: 305-311. 
Bradford, E.W., 1960. The dentine, a barrier to caries. Br. Dent. J., 109: 387-398.

Fish, E.W., 1933. An Experimental Investigation if Enamel, Dentine and the Dental Pulp. John Âale, Sons and Danielson, Ltd., London, pp: 427.

Gustafson, G., 1947. Microscopic examination of teeth as a means of identification in forensic medicine. J. Am. Dent. Assco., 35: 720-724.

Gustafson, G., 1950. Age determinations on teeth. J. Am. Dent. Assoc., 41: 45-54.

Harris, F.E., H.H. Mincer, M.K. Anderson and R.D. Senn, 2010. Age Estimation from Oral and Dental Structures. In: Forensic Dentistry, R.D. Senn and G.P. Stimson (Eds.). CRC Press, Taylor and Francis Group, LLC, New York, pp: 285.

Hodge, H.C., 1937. Micro hardness studies on transparent dentine. Br. Dent. J., 63: 181-192.

Isokawa, S., I. Satomura, T. Misu, T. Hasegawa and H. Honda, 1966. Some historadiographs of human dentine structures. J. Vihon Univ. Sch. Dent., 8: 191-197.

Johanson, G., 1971. Age determinations from human teeth. Odont. Revy, 21: 1-126.

Johnson, C.C., 1968. Transparent dentine in age estimation. Oral Surgery, 25: 834-838.

Manly, R.S. and E.J. Brooks, 1947. Transparency and light scattering of dental hard tissues. J. Dent. Res., 26: $427-434$.
Manly, R.S., J.F. Bonner and H.C. Hodge, 1940. Light scattering in normal human dentine. The calculation of absorption and scattering constants. J. Phys. Chem., 44: 745-751.

Miles, A.E.W., 1961. Ageing in the Teeth and Oral Tissues. In: Structural Aspects of Ageing, Bourne, G.H. (Ed.). Pitman, London, pp: 353-397.

Miles, A.E.W., 1972. Sans teeth: Changes in oral tissues with advancing age. Proc. R. Soc. Med., 65: 801-806.

Miles, A.E.W., 1976. Age Changes in Dental Tissues. In: Scientific Foundations of Dentistry, Cohen, B. and I.R.H. Kramer (Eds.). Vol. 29, William Heinemann Medical Books Ltd., London, pp: 363-375.

Nalbandian, J., F. Gonzales and R.F. Sïgnnaes, 1960. Sclerotic age changes in root dentine of human teeth as observed by optical, electron and x-ray microscopy. J. Dent. Res., 39: 598-607.

Simon, W.J. and W.D. Armstrong, 1941. Translucent dentine. J. Am. Dent. Assco., 28: 1115-1120.

Takuma, S. and S. Eda, 1966. Structure and development of the peritubular matrix in dentin. J. Dent. Res., 45: 683-692.

Vasiliadis, L., 1981. Root dentine translucency. Ph.D. Thesis, Bristol University, UK.

Vasiliadis, L., C. Stavrianos and P. Kafas, 2008. Assessment of dentin translucency in estimating age: A research. Int. J. Orofac. Sci., 1: 4-6. 